Jara L. ycols.

Rev. Chil. Pediatr. 66 (4); 186-191, 1995

\title{
Tiempos de la erupción dentaria temporal en pacientes con síndrome de Down
}

\author{
Lílian Jara S. '; Alejandro Ondarza G. ${ }^{2}$; Pamela Muñoz C. ${ }^{2}$; Rafael Blanco C. ${ }^{3}$
}

\section{Timing of deciduous dentition in patients with Down syndrome}

\begin{abstract}
Time anci sequence of deciduous dentilion were recorded among 255 Down syndiome palients at Santiago, Chile and compared to the normal chilecn population corresponding pattern. Boys with Down syndrome showed significontly deloyed dental eiuplion of raxillar right central ircisor $115.27 \pm 5.51 / 5$ months\} and right and left ateral incisors | $8.44 \pm 9.65 / 2$ and $: 8.13 \pm 10.01$ months respectivelyl and also in mandibular right central incisor $114.15 \pm 11.82$ morths and right and left canines $125.8 \% \pm 7.60 / 7$ and $26.65 \pm 7.43 \% 1$ months respectively: Girls with Down synerome showed significarit delays ir. the eruption ef even teeth: maxilar right and laft lcteral incisors $\{17.31 \pm 14.42$ and $17.31 \pm 14.42$ morihs ressecl veiyl, right and lef canires $130.70 \pm 6.45 \%$ 4 and $30.00 \pm 7.24 / 9$ mon'hs respectrvelyi cno first left molar $\{25.87 \pm 14.34$ months'; mandibular left central incisor ( $2.02 \pm 7.28 / 5$ months). rig-1 onc lefilatercl incisors $127.59 \pm 19.01$ and $24.00 \pm 23.36$ months respeclively, right and left camines $\{27.83 \pm 11.25$ and $25.80 \pm 10.00$ months respectively\} anc second igh molar $128.83 \pm 3545 ; 4$ months! The dental er uption sequence was simililar ariong Down syndrome ard normat children. With a few exceptions, no significant dexort.Jres from Goussian d'strioution were Found in 1רe oge of eruption omong both normal and Down syndrome individuals. The variances were significontly larger in Down syndrome cases.
\end{abstract}

(Key words: Down's syndrame, centilion, primary, tooth, deciduous.)

El sistema dentario es parte integral del cuerpo humano. Su crecimiento y desarrollo deben ser estudiados en paralelo con otros indicadores fisiologicos de madurez, como edad ósea, menarquia y peso. Uno de los criterios más usado para evaluar madurez dentaria es la erupción de las piezas correspondientes ${ }^{1}$.

En pacientes con cl síndrome de Down se ha descrito retardo en la erupción de ambas denticiones $^{2-17}$. En la dentición temporal, la erupción de los molares precede, en estos casos, a la de los incisivos y la serie de piezas temporales no sc completa antes de los cinco años ${ }^{18}{ }^{19}$. En una muestra de 48 niños australianos con síndrome de Down. las edades de erupción de piezas temporales fueron más tardías que en los normales,

1. Doctora en Ciencias. Departamento de Biología Celular y Genética, Facultad de Medicina Norte. Universidad de Chile.

2. Depattamento de Morfología Experimental. Facultad de Medicina, Utiversidad de Chile.

3. Deparlamento de Biología Celular y Genética, Facultad de Medicina Norte. Universidad de Chile. con excepción de los primeros y segundos molares en la maxila y de los segundos molares en la mandíbula. La erupción temporal en niños alemanes con trisomía 21 presentaba, en promedjo, seis meses de retraso, siendo el primer molar la primera o segunda pieza en emerger. La secuencia de erupción era muy variable en estos casos y no fue posible establecer diferencias de sexo o entre las hemiarcadas izquierda y derecha. Pocos autores han documentado observaciones controladas, con métodos estadísticos adecuados, sobre la cronología y secuencia de la dentición temporal en los pacientes con trisomía 21. El propósito del presente trabajo fue describir el patrón de erupción dentaria temporal en una muestra de niños chilenos con síndrome de Down y compararlo con el de la población chilena normal.

\section{Material y Métodos}

Los pacientes con síndrome de Down fueron seleccionados al azar entre la población escolar asistente a las es- 
cuelas especiales E-497. F-86, F-296, escuela diferencial E-469. Coocendes y Cindy College. 1odas de la ciudad de Santiago, Chile. La muestra incluyó a 255 iadividuos ( 128 mujeres) de 4 a 84 meses de edad. La fecha de nacimuento de los pacientes se obturo de los registros de la escuela respectiva.

El examen clfnico de la boca fue realizado por uno de los investigadores (PM), siguicado un procedimiento uniforme. bajo condiciones de buena iluminacion, consignan. do la presencia o ausencia de piezas dentarias. Una pieza dentaria se consideró erupcionada cuando cualquier porción de su corona era visible en la cavidad bucal.

Los pacientes fueron agrupados por sexo y edad, para to cual se definieron 14 grupos de edad en intervalos de 6 meses entre ellos. En cada grupo se registró número de dientes erupcionados correspondientes a cada pieza dentaría, así cono la proporción de dientes erupcionales en cada agrupación por edad, las que se transformaron en valorcs de probito usando un programa computacional. El análisis de probito es un método iterativo de aproximaciones sucesivas, que proporciona el mejor ajuste lineal por el procedimiento de los mínimos cuadrados. La edal pronedio de erupción, la desviación típica y el error típico se obtuvieror mediante el mismo prograuna computacional.

El tiempo de erupción de cada diente se comparó con un estudio para población normal realizado con el mismo thétodo de análisis ${ }^{\prime \prime}$. Para calcular la significación de las diferencias entre las edades de erupción en los niños con s, de Down en comparación con los normales se aplícó la prucba de Student con la correcieión de Welch. La prueba de $F$ se usó para comparar las varianzas para los tiempos de erupcion entre los pacientes de s. de Down y los niños nonnales y la prucba de chi para establecer el tipo de distribución de las edades de erupción en cada uno de los grupos analizados.

\section{Resultados}

En la tabla 1 se describe la distribución por edad y por sexo de la muestra. La tabla 2 presenta el promedio de edad de crupción en meses, la desviación típica y el error típico, para cada pieza dentaria temporal en niños y niñas. Los promedios cuyos errores típicos fucron muy grandes no se consideraron en los análisis posteriores.

Los resultados de la prueba de chi ${ }^{2}$ permitieron establecer que, exceptuando la pieza 5,4 en mujeres, las edades de erupción presentaron una distribución gaussiana en los individuos normales y en el grupo Down. Los resultados de la prueba de $\mathrm{F}$ indicaron que las varianzas existentes en los niños con s. de Dawn son significativamente superiores a las de los normales, siendo la única excepción la pieza 8,5 en mujeres, en donde las varianzas fueron similares.
En la tabla 3 se presenta la edad de la erupcion dentaria temporal en niños y niñas con síndrome de Down y en la tabla 4 la de la población chilena normal. En las niñas con $s$. de Down ésta se inició con la emergencia del incisivo central inferior derecho a los 7,51 meses, la pieza siguiente fue el incisivo central inferior izquierdo a los 12,02 meses, luego apareció el primer molar superior derecho a los 13,28 meses y en cuarto lugar el incisivo central superior izquierdo a los 15 o 16 meses. En los varones $\mathrm{cl}$ proceso comenzó a los 7,57 meses con el incisivo lateral inferior derecho, seguido por el incisivo lateral inferior izquierdo a los 9,31 meses, luego el incisivo central superior izquierdo a los 9,77 meses y en cuarto lugar el incisivo central inferior izquierdo a los 11,07 meses.

La cronología del proceso eruptivo de piezas temporales en los niños y niñas con s. de Down es diferente que en la población normal. existiendo, además, diferencias entre niñas y niños con la anomalía cromosómica. En efecto, en los niños con síndrome de Down se registró retraso significativo en la erupción de seis piezas dentales (tres superiores y tres inferiores): el incisivo central derecho (15,27 $\pm 5,515$ meses) y los incisivos laterales derecho e izquierdo $(18,44 \pm 9,652$ y $18,13 \pm 10,01$ meses respectivamente) en el maxilar superior; cl incisivo central derecho $(14,15 \pm 11,82$ meses $)$ y los caninos derecho e izquierdo $(25,87 \pm 7,667$ y $26,65 \pm$

\section{Tabla 1}

Distribución por edad y sexo en una muestra de población escolar chilena con síndrome de Down

\begin{tabular}{cccc}
\hline Edad (meses) & Varones & Mujeres & Ambos sexos \\
\hline $0-6$ & 3 & 2 & 5 \\
$7-12$ & 6 & 7 & 13 \\
$13-18$ & 6 & 6 & 12 \\
$19-24$ & 8 & 7 & 15 \\
$25-30$ & 12 & 11 & 23 \\
$31-36$ & 9 & 13 & 22 \\
$37-42$ & 11 & 11 & 22 \\
$43-48$ & 8 & 14 & 22 \\
$49-54$ & 18 & 13 & 31 \\
$55-60$ & 11 & 10 & 21 \\
$61-66$ & 7 & 8 & 15 \\
$67-72$ & 10 & 6 & 16 \\
$73-78$ & 11 & 10 & 21 \\
$79-84$ & 7 & 10 & 17 \\
\hline Total & 127 & 128 & 255 \\
\hline
\end{tabular}




\section{Tabla 2}

Promedios, desviaciones típicas y enrores típicos en meses.

para la erupción dentaria temporal según sexo en una muestra de población escolar chilena con síndrome de Down

\begin{tabular}{|c|c|c|c|c|c|c|c|c|c|}
\hline HSD & Sexo & $\overline{\mathbf{x}}$ & DT & ET & HSI & Sexo & $\overline{\mathbf{x}}$ & DT & ET \\
\hline & V & 27,66 & 5.645 & 2,042 & $\mathrm{v}$ & & 27.66 & 5.645 & 2,042 \\
\hline \multirow[t]{3}{*}{ M2 } & & & & & $\mathrm{M} 2$ & & & & \\
\hline & $M$ & 29.12 & 6.067 & 1,838 & & M & 29.60 & 5.649 & 1,637 \\
\hline & $\mathrm{V}$ & 17.21 & 3.611 & 1,499 & & $v$ & 17,21 & 3,611 & 1,499 \\
\hline \multirow[t]{3}{*}{ Mi } & & & & & Mi & & & & \\
\hline & $\mathbf{M}$ & 13.28 & - & - & & $\mathrm{M}$ & 25.87 & 14.340 & 5.253 \\
\hline & $v$ & 12,02 & 32,100 & 19,480 & & V & 22,16 & 13,850 & 8.260 \\
\hline \multirow[t]{3}{*}{$\mathrm{C}$} & & & & & $\mathrm{C}$ & & & & \\
\hline & $\mathbf{M}$ & 30.70 & 6,454 & $1.44 ?$ & & $\mathbf{M}$ & 30.60 & 7,249 & 1,586 \\
\hline & V & 18,44 & 9,652 & 2,424 & & $\mathrm{~V}$ & 18,13 & 10,010 & 3,422 \\
\hline \multirow[t]{3}{*}{ IL } & & & & & $\mathrm{L}$ & & & & \\
\hline & $M$ & 17.31 & 14,420 & 3,625 & & M & 17,31 & 14,420 & 3.625 \\
\hline & V & 15.27 & 5,515 & 1,797 & & v & 9,77 & 30,620 & 10,250 \\
\hline \multirow[t]{2}{*}{ IC } & & & & & IC & & & & \\
\hline & $\mathrm{M}$ & 15,16 & 9,340 & 4.778 & & M & 15,16 & 9,340 & 4,778 \\
\hline \multirow[t]{2}{*}{ HID } & Sexo & $\overline{\mathbf{x}}$ & DT & $\mathbf{E T}$ & HII & Sexo & $\overline{\mathbf{x}}$ & DT & $\mathbf{E T}$ \\
\hline & $v$ & 14,15 & 11,820 & 3.909 & & $\mathrm{v}$ & 11,07 & 10.760 & 4.288 \\
\hline \multirow[t]{3}{*}{ IC } & & & & & IC & & & & \\
\hline & $M$ & 7,515 & 17,750 & 16,020 & & $\mathbf{M}$ & 12,02 & 7.286 & 2.699 \\
\hline & V & 7,569 & 35.240 & 13,750 & & $\mathbf{M}$ & 9,313 & 30,610 & 11.570 \\
\hline \multirow[t]{3}{*}{ IL } & & & & & $\mathrm{lL}$ & & & & \\
\hline & $\mathbf{M}$ & 27.59 & 19.010 & 3,976 & & M & 24,66 & 23,860 & 5,447 \\
\hline & V & 25.87 & 7.667 & 2,046 & & V & 26.65 & 7.431 & 1,906 \\
\hline \multirow[t]{3}{*}{$\mathrm{C}$} & & & & & $c$ & & & & \\
\hline & $\mathbf{M}$ & 27.81 & 11,250 & $2,6.33$ & & $M$ & 28,80 & 10,600 & 2,360 \\
\hline & $\mathrm{V}$ & 17.21 & 3,611 & 1,498 & & $\mathrm{v}$ & 17,88 & $5 . J 6 \theta$ & 1,930 \\
\hline \multirow[t]{3}{*}{ M1 } & & & & & MI & & & & \\
\hline & M & 57.38 & $-12,740$ & $146,180^{*}$ & & $M$ & -322.40 & 262,170 & 2939,200 \\
\hline & $\mathrm{V}$ & 27.71 & 5.562 & 1,487 & & $v$ & 27.71 & 5,562 & 1,487 \\
\hline \multirow[t]{2}{*}{ M2 } & & & & & M2 & & & & \\
\hline & $\mathrm{M}$ & 28,83 & 3,454 & 1.127 & & M & $32.3 \mathrm{I}$ & 13.430 & 4.382 \\
\hline
\end{tabular}

* : no considerado para análisis posteriores debido a su gran error típico.

** : datos no ajustados a la distribución gaussiana.

HSD: hemiarcada superior derecha

HSI: hemiarcada superior izquierda

HID: hemiarcada inferior derecha

HII: hemiarcada inferior izquierda

\author{
DT: desviación típica \\ ET: error típico \\ $\bar{x}$ : promedio \\ $\checkmark:$ varones \\ $\mathrm{M}:$ mujeres
}

IC: incisivo central

IL: incisivo lateral

$\mathrm{C}$ : canino

MI: primer molar

M2: segundo molar 
Tabla 3

Secuencia cronológica de erupción de la dentición temporal en una muestra de población escolar chilena con síndrome de Down*

\begin{tabular}{|c|c|c|c|c|c|c|c|c|c|c|c|}
\hline HSD & $\overline{\mathbf{x}}$ & DT & HSI & $\begin{array}{c}\text { Varones } \\
\overline{\mathbf{x}}\end{array}$ & DT & HSD & $\overline{\boldsymbol{x}}$ & DT & HSI & $\underset{\overline{\mathbf{x}}}{\text { Mujeres }}$ & DT \\
\hline C & 12,02 & 32,10 & IC & 9,77 & 30.620 & MI & 13,28 & 20,440 & IC & 15,16 & 9,340 \\
\hline IC & 15,27 & 5.515 & $\mathrm{Ml}$ & 17.21 & 3.611 & IC & 15,16 & 9,340 & IL & 17,31 & 14.420 \\
\hline M1 & 17,21 & 3.611 & IL & 18,13 & 10,010 & $\Pi$ & 17.31 & 14.420 & MI & 25,87 & 14,340 \\
\hline IL & 18.44 & 9.652 & $c$ & 22,16 & 13,850 & $\mathrm{M}_{2}$ & 29,12 & 6,067 & $M 2$ & 29,60 & 5,649 \\
\hline $\mathrm{M} 2$ & 27.66 & 5.645 & M2 & 27,66 & 5,645 & C & 30,70 & 6.454 & $c$ & 30,60 & 7.249 \\
\hline HID & $\overline{\mathrm{x}}$ & DT & HII & $\overline{\mathbf{x}}$ & DT & HID & $\overline{\mathbf{x}}$ & DT & HII & $\overline{\mathbf{x}}$ & DT \\
\hline IL & 7.569 & 35,240 & IL & 9,313 & 30,610 & IC & 7,515 & 17.75 & IC & 12.02 & 7,286 \\
\hline IC & 14,150 & 11,820 & IC & 11,070 & 10,760 & L & 27.590 & 19.01 & IL & 24.66 & 23,860 \\
\hline MI & 17,210 & 3,611 & MI & 17,880 & 5,166 & C & 27.830 & 11.25 & C & 28,80 & 10,600 \\
\hline C & 25,870 & 7,667 & C & 26,650 & 7,431 & M2 & 28,830 & $3,4 \$ 4$ & M2 & $32,3]$ & 13,430 \\
\hline $\mathbf{M} 2$ & 27,310 & 5,562 & $\mathrm{M} 2$ & 27,710 & 5,562 & MI & 57,380 & $-32,740$ & MI & $-322,40$ & 262,170 \\
\hline
\end{tabular}

* edad en meses

HSD: hemiarcada superior derecha

HSI: hemiarcada superior izquierda HID; hemiarcada inferior derecha HIl: hemiarcada inferior izquierda DT: desviación típicn $\bar{x}$ : promedio
IC: incisivo central

IL: incisivo latera]

C : canigo

M1: primer molar

M2: segundo molar

Tabla 4

Secuencia cronológica de erupción de la dentición temporal en una muestra de población escolar chilena normal**

\begin{tabular}{|c|c|c|c|c|c|c|c|c|c|c|c|}
\hline HSD & $\overline{\mathbf{x}}$ & DT & HSI & $\begin{array}{l}\text { Varones } \\
\frac{\bar{x}}{4}\end{array}$ & DT & HSD & $\overline{\mathbf{x}}$ & DT & HSI & $\frac{\text { Mujeres }}{\overline{\mathbf{x}}}$ & DT \\
\hline IC & 9,499 & 2,546 & IC & 9,305 & 2,410 & IC & 9,359 & 3.114 & $\mathrm{JC}$ & 9.340 & 2,854 \\
\hline IL & 10.510 & 2,430 & IL & 9.860 & 3.587 & 느 & 10.400 & 3.178 & L & 10,300 & 3.192 \\
\hline $\mathrm{MI}$ & 15,430 & 1,961 & MI & 15,290 & 2.033 & MI & 15.520 & 2,042 & M1 & 15.890 & 2,210 \\
\hline C & 18,360 & 3.215 & $C$ & 18,140 & 3,272 & $c$ & 18.670 & 3,105 & C & 38,700 & 3,099 \\
\hline M2 & 26,870 & 3,931 & $M 2$ & 26,630 & 3,952 & M2 & 26,980 & 3,614 & $\mathrm{M} 2$ & 27,050 & 3.502 \\
\hline HID & $\overline{\mathbf{x}}$ & DT & HII & $\overline{\mathbf{x}}$ & DT & HID & $\overline{\mathbf{x}}$ & DT & HII & $\overline{\mathbf{x}}$ & DT \\
\hline IC & 6.370 & 1,180 & IC & 6,370 & 1.180 & IC & 6.390 & 1,460 & IC & 6.390 & 1.460 \\
\hline IL & 12.510 & 3.439 & IL & 12.900 & 3,982 & IL & 12,770 & 3,412 & IL & 12,490 & 3.450 \\
\hline M1 & 15.880 & 2.078 & M1 & 15.860 & 2,160 & M1 & 15,950 & 2.033 & M1 & 16,070 & 2,394 \\
\hline C & 19.040 & 3.377 & C & 18.980 & 3,337 & $\mathrm{C}$ & 19,230 & 3,153 & C & 19,080 & 3,373 \\
\hline M? & 26.140 & 3.588 & $\mathrm{M} 2$ & 26.110 & 3,724 & M2 & 26,280 & 3,725 & M2 & 26.280 & 3.529 \\
\hline
\end{tabular}

* edad en meses; +datos de Palomino H. y col.: Odontología Chilena 1908; 28 (123-124): 73-77.

HSD: hemiarcada superior derecha HSI: hemiascada superior izquierda HID: bemiarcada inferior derccha HII: hemiarcada inferior izquierda DT: desviación tipica $\overline{\mathrm{x}}$ : promedio
IC: incisivo central

IL. incisivo lateral

C. canino

MI : primer molar

M2: segundo molar 
7,431 meses, respectivamente) en el maxilar inferior. En las mujeres con $\mathrm{s}$. de Down se registró retardo en la erupción de 11 piezas (cinco superiores y seis inferiores): los incisivos laterales derecho e izquierdo $(17.31 \pm 14.42$ y $17,31 \pm 14,42$ meses, respectivamente), los caninos derecho e izquierdo $(30,70 \pm 6,454$ y $30,60 \pm 7,249$ meses, rcspectivamente, y el primer molar izquierdo $(25,87 \pm 14,34$ meses $)$ en el maxilar superior; el incisivo central izquierdo $(12,02 \pm 7,286$ meses $)$, los incisivos laterales derecho e izquierdo $(27,59 \pm 10,01$ y $24,66 \pm$ 23.86 meses, respectivamente), los caninos derecho e izquierdo $(27,83 \pm 11,25$ y $28,80 \pm$ 10,60 mescs, respectivamente) y el segundo molar derecho $(28,83 \pm 3,454$ meses $)$ en el maxilar inferior.

\section{Comentario}

Existe un retardo estadísticamente significativo en la erupción de piezas deciduales cspecíticas en los pacientes con s. de Down. Si bien estos resultados concuerdan con los obtenidos por otros autores, no son comparables con cllos debido a las diferentes metodologías empleadas. La prueba do probito, aplicada en este estudio, es el método estadístico más adecuado para el análisis de la erupción dentaria.

Tanto en niñas como en niños chilenos con s. Down la erupción decidual comenzó a los 7,5 meses, 10 que no representa una diferencia temporal importante con respecto al inicio del proceso cruptivo normal (6.3 meses), en discrepancia con los resultados obtenidos en niños caucasoides (alemanes) con la misma anomalía, en quienes la emergencia de piezas temporales presenta en promedio seis meses de retraso ${ }^{20}$. Además, en los niños chilenos de ambos sexos con s. de Down, las primeras piezas en aparecer fueron los incisivos, mientras en otras experiencias son los molares ${ }^{7} \cdot 13,20$. Las diferencias observadas en Ia cronología y la secuencia del proceso eruptivo decidual pudiesen deberse a diferencias étnicas o metodológicas.

La secuencia de la erupción de la dentadura decidual no resultó completamente diferente en los niños con $s$. de Down que en la población normal. En niños nomales las alteraciones de la secuencia son más frecuentes entre quienes sufren retrasos en la erupción, que cuando ésta se adelanta u ocurre dentro de los márgenes normales de liempo.

La mayoría de los informes describen gran variación en los tiempos de aparición de la dentadura decidual en los niños con s. de Down ${ }^{7}{ }^{10,20}$, con lo que concuerdan nuestros resultados, con excepción del primer molar superior derecho en mujeres. La mayor varianza en e) proceso enuptivo es también observable en otras características de estos pacientes ${ }^{10}$. Hasta el momento no se conocen las causas del retraso en la dentición temporal en los niños con $\mathrm{s}$. de Down, esencialmente porque tampoco se tiene suficiente informacion sobre los factores que controlan el proceso eruptivo normal. Durante la erupción ocurre reabsorción osea, proceso que parecería estar deprimido en los niños con trisomía ${ }^{22}$. Algunos hechos sugieren que la velocidad del proceso eruptivo estaría influida por las casacterísticas de la vascularización del tejido conectivo periradicular ${ }^{7}$. La disminución de la circulación periférica podría ser un factor coadyuvante en el retardo eruptivo. Las raíces de los dientes en los pacientes con s. de Down son cortas ${ }^{2.3}$, pero no está claro si ello influye en la erupción. El retraso en la aparición de los dientes deciduales podría ser parte del retraso general del crecimiento y del desarrollo que caracteriza al sindrome. El crecimiento de ambos maxilares está retrasado en estos casos. Algunos autores han correlacionado el bajo peso de nacimiento con retardo de la erupción temporal ${ }^{2427}$.

\section{Resumen}

Se examinaron 255 pacientes con síndrome de Down que asisten a escuelas especiales de Santiago, Chile, con el propósito de describir los tiempos de erupción para la dentición decidual y compararlos con los de la población normal. En los niños con síndrome de Down se observa retraso significativo en la erupción de los siguientes dientes: $\mathrm{e} 1$ incisivo central derecho ( $15,27 \pm 5,515$ meses) y los incisivos laterales derecho e izquierdo $(18,44 \pm 9,652$ y 18,13 $\pm 10,01$ meses respectivamente) en el maxilar superior: el incisivo central derecho $(14,15 \pm$ 11,82 meses) y los caninos derecho e izquicrdo $(25,87 \pm 7,667$ y $26,65 \pm 7,431$ meses, respectivamente) en el maxilar inferior. Las niñas con síndrome de Down presentan retraso significati- 
vo en la erupción de los incisivos laterales derecho e izquierdo $(17,31 \pm 14,42$ y $17,31 \pm 14,42$ meses, respectivamente), los caninos derecho e izquierdo $(30,70 \pm 6,454$ y $30,60 \pm 7,249$ meses, respectivamente, y el primer molar izquierdo $(25,87 \pm 14,34$ meses) en el maxilar superior; el incisivo central izquierdo $(12,02 \pm 7,286$ meses), tos incisivos laterales derecho e izquierdo $(27,59 \pm 10,01$ y $24,66 \pm 23,86$ meses, respectivamente), los caninos derecho e izquierdo $(27,83 \pm 11,25$ y $28,80 \pm 10,60$ meses, respectivamente) $y$ el segundo molar derecho $(28,83 \pm$ 3,454 meses) en el maxilar inferior. La secuencia de erupción en los niños con $s$. de Down fue similar a la observada en los normales. Las edades de erupción mostraron una distribución gaussiana y las varianzas existentes en el grupo con $\mathrm{s}$. de Down fueron significativamente superiores a las de los normales.

(Palabras clave: síndrome de Down, dentición primaria, dientes deciduales, erupción.)

\section{Referencias}

1. Demirjian A. Falkner F. Tanner M: Human Growth. Isı. ed. Plenum Press. New York, 1978: 413-441.

2. Oster J: Mongolism. A clinicogenealogical investigation comprising 526 mongols living on Seeland and neighboring islands in Denmark, Copenhagen. Danish Science Press Ltd. 1953.

3. Spizer $R$, Quilian $R L$ : Observations on congenital auomalies in teeth and skull in two groups of mental defectives. Brit J Radiol 1958: $31: 596.598$.

4. Cohen MM, Winner RA: Dental and facial characteristhics in Down's syndrome (mongolism). I Dent Res 1965: 44: 197-208.

5. Burkia DH: Ages of eruption of permanent teeth in mongols. J Ment Defic Res 1966; 10: 190-197.

6. Otero $E$. Sznodjer $N$ : Agenesia de dientes permanentes en el síndrome de Down (mongolismo). Rev Asoc Argent 1966; 54: 257-259.

7. Roche $A F$, Barkla $D H$ : The development of the dentition in mongols. Aus Dent J 1967: 12: 12-16.

8. Spitzer $R$ : Observations on congenital dentofacial disorders in mongolism microcephaly. O.S. O.M. \& O.P. 1967; 24: 325-332.

9. Ornes $G$ : Congenitally absent permanent teeth anong mongols and their sibs. J Ment Defic Res 1971: IS: 291-302.
10. Cohen $M$. Sr. Cohen $M M J r$ : The oral manifestations of trisomy $G_{1}$ (Down syodrome). Birth Defects 1971; 7: $241-251$.

11. Orner $G$ : Eruption of the permanedt teeth in mon. goloid children and their sibs. J Deat Res 1973; 52: 1202-1208.

12. Orner $G$ : Posteruptive toath age in children with Down's syadrome and their sibs. J Dent Res 1975: 54: 581-587.

13. Molina I, Viñas L. Gacía-Godoy FM: Eropción clínica de los dientes permanentes en niflos con impedimentos mentales de Santo Domingo. Acta Odontol Pediatr 1982; 3: 69-73.

14. Garcia $B C$, Mas BC y Pérez FD: Una aportación at estudio de las caries en escolares españoles afectados de trisomia 21. Avances en Odontoestomarología $1985 ; 1: 137-146$.

15. Jara $L$, Ondarza A. Infante $I I$ et al.: Anomalias orofaciales en pacientes con síndrome de Down en una muestra de población chilena. Rev Chil Pediatr 1986; 57: 510-513.

16. Palomino H. Blanco R. Cisternas A: Edad y orden de aparición de los dientes deciduales en una población de Santiago. Odontol Chilena 1980; 28: 73-77.

17. Jara L. Ondarza A, Blanco R: Cronología de la erupción dentaria permanente es pacientes con sf́ndrome de Down. Rev Chil Pediatr 1992: 63: 89-95.

18. Levitson A, Friedman A, Stamps F: Variability of mongolism. Pediatrics 1955; 16: 43-53.

19. Shroff $F R$ : Identification by teeth. A possible source of error. Br DenI J 1959; 107: 178-180.

20. Fischer-Brandies $H$ : Über den Zeitpunkt des Durchbruchs der Milchzähne bei Morbus Down. Fortschr. Kieferorthop. 1989; 50: 144-151.

21. Robinov $M$, Richards $T$. Anderson $M$ : The eruption of deciduous teeth. Growth 1942; 6: 127-133.

22. Roche A, Seward $F$, Sunderland $S$ : Nonmetrical observations on cranial roentgenograms in mongolism. Amer J Roentgenol 1961; 85: 659-662.

23. Mac Millan RS. Kashgarian M: Relation of human abnormalities of structure and function to abnormalities of the dentition. Mongolism. JADA 1961; 63: 368-373.

24. Billewicz WZ: A note on estimation of calendor age on the basic of developmeat of primary teeth. J Trop Pediatr Enviton 1973; 19: 243-245.

25. Infante $P F$, Owen GM: Relation of chronology of deciduous tooth emergence to height, weight and head circumference in children. Arch Oral Biol 1973; 18: 1411-1412.

26. Trupkin DP: Eruption pattems of the first primary toolb in infants who were underweight at birth. J Dent Child 1974; $41: 279$.

27. Delgado $H$, Habitch JP. Yarbrough $C$, et al.: Nutritional status and the timing of deciduous tooth enuption. Am J Clin Nutr 1975: 28: 261. 